\title{
Spatial coding of auditory signals
}

\author{
LLOYD R. PETERSON, JACK HOLSTEN, and PETER SPEVAK \\ Indiana University, Bloomington, Indiana 47401
}

\begin{abstract}
Sequences of nine binary auditory signals (dots and dashes) were presented to 20 subjects in Experiment I. The subjects were instructed to internally organize the signals into two-dimensional arrays. Visual patterns (letters) could be recognized in these imaginary arrays in both upright and rotated orientations. In Experiment II, a group of nine subjects, which was instructed to use spatial imagery of this kind, reproduced significantly longer sequences of signals than nine control subjects. By means of internal spatial organization, experimental subjects were able to reproduce sequences up to 45 signals in length, whereas control subjects recalled near the chance level for portions of sequences longer than nine signals. Three levels of information processing were postulated to account for the results, with spatial organization occupying a mediating level between acoustic and verbal levels.
\end{abstract}

In recent years progress has been made in obtaining evidence other than that provided by introspection from which inferences concerning subjective imagery can be made. One kind of evidence is the enhanced learning rate found after instructions to use imagery (Bower, 1972). Other evidence can be found by relating imagery ratings of words to difficulty of learning lists of varying degrees of rated imagery-value (Paivio, 1971). Further evidence comes from interference by a concurrent task utilizing the same sensory mode as that assumed for the instructed imagery (Atwood, 1971).

The present experiments arose out of a desire to establish an experimental technique for the study of imagery which would provide fairly precise control over imagery used by subjects, and convince skeptics that imagery of the assumed kind was indeed being used by the subjects. To achieve these objectives, experiments were designed to investigate the construction of spatial imagery from sensory information that is not normally the basis for spatial judgments. Binary signals in the auditory mode were presented to subjects with instructions to organize them in such a way that two-dimensional spatial patterns could be identified. The formation of maps from verbal instructions has been reported (Bower, 1972); the present studies utilized nonverbal inputs. Like nonsense syllables, the individual elements of the auditory message were familiar, but the pattern was not. Unlike nonsense syllables, the meaning of the set of elements could be discovered by the application of a simple spatial grammar. This spatial grammar was taught by instructions and practice tests.

The subjects were instructed to organize auditory signals into a pattern which could be identified as a letter. The subject sat with eyes closed scanning an imagined two-dimensional field, a slow-motion analogue of a picture being built up on a television tube. The subject was told to imagine a three by three array of lights, and a sequence of binary signals indicated whether successive positions contained lights which were

This research was facilitated by NSF Grant GB 7947 to Indiana University, with the senior author as principal investigator.

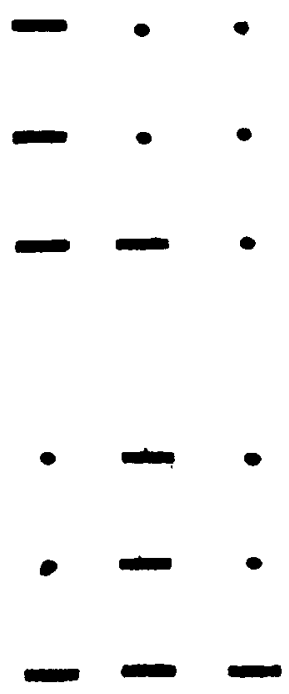

Figure 1. Four examples of matrix organization of auditory signals. (An " $L$ " is shown in upright or 0-deg rotation, " $Y$ " in 90-deg rotation, " $T$ " in 180-deg rotation, and " $V$ " in 270-deg rotation.)

on (dash) or off (dot). The lighted positions formed a stylized pattern representing a letter of the alphabet, as illustrated in Figure 1.

The initial experiment tested ability to identify letters of the alphabet in various orientations. A second experiment required subjects to use the spatial patterns as mnemonic aids in reproducing binary sequences of various lengths.

\section{EXPERIMENT I}

\section{Method}

Twenty students from introductory psychology classes at Indiana University, Bloomington, served as subjects in order to fulfill a class requirement. They were tested in groups of two, three, or four, each subject being shielded from the others by wooden screens. Earphones minimized extraneous sounds and supplied a uditory signals. Sequences of signals were tape-recorded from the output of three tone generators and a white noise generator. Programming of the signals was accomplished by means of a PDP-8L digital computer. 
Tape-recorded instructions called attention to a $76 \times 127 \mathrm{~mm}$ card displaying a three by three array of dots. The subjects were instructed to close their eyes and imagine a similar display of lights. They were told that a brief burst of noise $(70 \mathrm{~ms})$ would indicate that the light in a given position in the array was off, whereas a tone $(288 \mathrm{~Hz}$ for $140 \mathrm{msec})$ audible over the noise would signal that the light was on. Double tones (410 and $492 \mathrm{~Hz}$ sounding together for $1.6 \mathrm{sec}$ ) would serve as a ready signal. The silent interval between two signals in a sequence was $.7 \mathrm{sec}$ save at the end of a group of three signals when $1.4 \mathrm{sec}$ elapsed before the beginning of the next group.

Four practice tests were given using sequences of dots and dashes that described the letters $I, H, X$, and $Z$. These letters were not used in the experiment proper. A matrix of dots on a sheet of paper provided the opportunity for responding. The instructions were to write the letter that was identified if possible, otherwise to circle the dots which indicated the position of lights that were on. The correct letter was given by voice over earphones toward the end of a 19-sec interval separating the end of one test from the onset of the ready signal for the next test.

Next, subjects were given four tests in which the auditory signals for T, L, V, and Y were presented. Again subjects marked down the appropriate letter if they identified it. Otherwise they marked the positions of dashes (light on). However, no knowledge of results was given on these tests, nor were these letters in the experimental set ever mentioned by name by the experimenter at any time during the experiment.

An answer sheet displaying the letters of the alphabet was then provided, and subjects were told to mark the letter they identified. They were told to expect some letters in the next tests to be in rotated orientations, in addition to those which would be upright. Sixteen tests followed in which auditory signals describing each of the four letters ( $T, L, V, Y)$ were presented in each of four positions resulting from rotations counterclockwise of $0,90,180$, or $270 \mathrm{deg}$. Within a block of four tests each letter occurred once, and all four orientations were tested.

\section{Results}

On their first test using auditory signals describing each of the letters in the experimental set, subjects wrote the correct letter on .79 of the tests $(\mathrm{SE}=.06)$. In the other tests, the letter " $V$ " accounted for most of the failures of identification. Note that not only were the sequences of auditory signals novel to the subjects, the subset of the alphabet that would be used was unknown. The appropriate letters could only be discovered by assigning the auditory signals to positions in a three by three array according to the spatial rules.

In the next 16 tests in which spatial rules produced letters in four orientations, subjects achieved an even higher degree of accuracy. The mean proportions correct were $.95, .92, .92$, and .92 (SE $=.02, .04, .04$, and .04$)$ for the 0-, 90-, 180-, and 270-deg rotations, respectively. Orientation was not a significant factor in an analysis of variance based on arcsin transformations of proportions correct per subject, $F(3,76)<1$. Differences among individual letters were trivial.

Note that subjects had not heard individual sequences of signals before in this portion of the experiment save in the case of the upright orientation. Thus, in $75 \%$ of these tests, they were identifying a letter in a novel sequence of binary auditory signals, a striking demonstration of the efficiency of the coding performed by the subjects.

\section{EXPERIMENT II}

A second experiment investigated the capacity of subjects to utilize spatial organization as a mnemonic device in the reproduction of long sequences of binary signals. The subjects were instructed to reproduce sequences of from 9 to 45 signals by pressing two keys on a Teletype. Experimental subjects were instructed to identify a letter in each group of 9 signals by spatial organization of the signals. Control subjects attempted the same reproductions without having the instructions relating to spatial imagery.

\section{Method}

The population from which subjects in Experiment II were sampled was similar to that of Experiment I, save that the experiment was conducted over a year later. Eighteen subjects were processed one at a time in a $1.77 \times 2.28 \mathrm{~m}$ room in which a subject was seated before a Teletype interfaced with a PDP-8L computer in an adjacent room. Auditory signals were presented over earphones as the computer programmed them, and subjects responded by typing, although no visible record of their typing was seen by the subjects.

In Stage 1, all subjects were given eight practice tests on patterns of dots and dashes which were not used later in the experiment proper. The subjects attempted to reproduce sequences of nine binary signals. Presentations were similar to those described in Experiment I, save that a bell indicated the end of a presentation. The response alternatives were (a) pressing a key labeled with a dot, or (b) one labeled with a dash. By pressing the space bar, labeled "FINISH," subjects indicated the end of their sequence of responses on a particular test. About $5 \mathrm{sec}$ after pressing the FINISH bar, the next test was initiated by a double tone as a ready signal.

In Stage 2, subjects were given differential treatment, 9 being randomly assigned to the experimental group and given imagery instructions adapted from those of Experiment I. These subjects were told to call out the letter they recognized in the spatial array and then reproduce the sequence. The remaining nine subjects, constituting the control group, were simply told to reproduce the series of dots and dashes. Both groups were then given 12 tests on sequences that could be organized into patterns of the letters $\mathrm{L}, \mathrm{C}, \mathrm{T}, \mathrm{X}, \mathrm{V}$, or $\mathrm{Y}$ in one of the four rotations described in the previous experiment.

Finally, in Stage 3, subjects were given 10 tests in which 5 lengths of sequence were each tested twice. The number of signals in a given sequence was either $9,18,27,36$, or 45 dots and dashes. Each group of 9 could be organized into a representation of one of the letters from Stage 2. A pause of $1.2 \mathrm{sec}$ was inserted between groups of 9 signals, and the bell signaled the end of the presentation and the beginning of the response period. The subjects in the imagery group were told sequences might involve several letters, while subjects in the control group were told that there might be several groups of 9 . In both cases, subjects were told to reproduce as much of the sequence as possible.

A single random order of tests was used for all subjects during the typing practice of Stage 1. In Stages 2 and 3, three random orders of tests were used in each stage over the group. No knowledge of results was given after any of the tests. 


\section{Results}

The subjects given imagery training were found to be markedly superior to control subjects in recalling sequences of signals longer than nine. The accuracy of reproduction of signals in Stage 3 is summarized in Figure 2. In this figure and in the analysis of variance to be described later, the proportion of signals correctly typed on each test was calculated for each subject, and each point on the graph is the mean of these proportions. There is little difference in accuracy of recall for sequences of only nine signals, with both imagery and control subjects being close to errorless performance. However, there is only a moderate decrease in accuracy of recall for the imagery subjects as the sequence lengthens, while performance for the control subjects falls to near the chance level for sequences longer than nine.

It might be presumed that .5 is the appropriate chance level in this experiment, but this is reasonable only to the extent that the correct number of responses occurred. Not all subjects typed enough signals on each test. One subject in the imagery group did not respond on any test with more than nine keypresses prior to the FINISH press, and there is no indication of his having used imagery. In general, however, subjects instructed in the use of imagery tended to give the appropriate number of signals, while control subjects had greater difficulty in keeping track of the total number of signals. This explains why proportion correct drops below .5 for the longer sequences of the control subjects

Proportions correct were subjected to an arcsin transformation preparatory to an analysis of variance. Instructions (imagery vs control) were significant, $F(1,16)=18.94, p<.01$. Number of signals was also significant, $F(4,64)=25.74, p<.01$. First half vs second half (of Stage 3 ) was not significant, $F(1,16)=2.63$, $p>.05$. The interaction between number of signals and instructions was significant, $\mathrm{F}(4,63)=5.23, \mathrm{p}<.01$. Other interactions were not significant.

An analysis of serial position effects for blocks of nine signals is summarized in Table 1. Note that when sequences of signals longer than nine were presented to

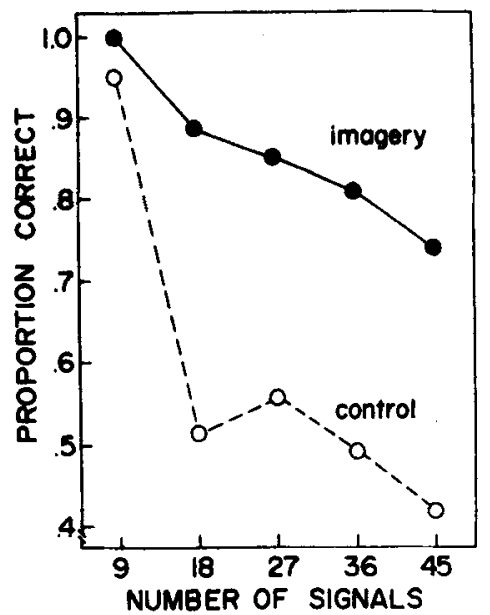

Figure 2. Proportions of signals correctly recalled in Experiment II by two groups of subjects. Standard enors for the imagery group were $.02, .08, .09, .10$, and .11 ; for the control group they were $.05, .12, .12, .12$, and .12 .

control subjects, they did reasonably well at recalling the initial group of nine, reproducing accurately about $75 \%$ of the signals. However, signals beyond the first group were less frequently recalled correctly, if, indeed, responses occurred at all. Control subjects may have adopted the strategy of rehearsing the initial group even though it meant disregarding subsequent signals. Since the length of the sequence was uncertain, this was not an unreasonable strategy.

A primacy effect, though of lesser magnitude, can also be seen in the responding of the imagery subjects. Errors were more numerous with each additional set of nine signals in a sequence. No recency effect is discernable, this being understandable in the light of some failures of subjects to make enough responses.

\section{DISCUSSION}

The data from Experiments I and II strongly support the interpretation that most subjects who were instructed to use imagery organized sequences of

Table 1

Mean Proportions Correct and Standard Errors by Block of Nine: Experiment II

\begin{tabular}{|c|c|c|c|c|c|c|c|c|c|c|c|}
\hline \multirow{2}{*}{\multicolumn{2}{|c|}{$\begin{array}{c}\text { Sequence } \\
\text { Length }\end{array}$}} & \multicolumn{2}{|c|}{1} & \multicolumn{2}{|c|}{2} & \multicolumn{2}{|c|}{3} & \multicolumn{2}{|c|}{4} & \multicolumn{2}{|c|}{5} \\
\hline & & Mean & SE & Mean & SE & Mean & SE & Mean & SE & Mean & SE \\
\hline $\begin{array}{l}\text { Imagery } \\
\text { Group }\end{array}$ & $\begin{array}{r}9 \\
18 \\
27 \\
36 \\
45\end{array}$ & $\begin{array}{l}.99 \\
.99 \\
.93 \\
.92 \\
.94\end{array}$ & $\begin{array}{l}.00 \\
.01 \\
.04 \\
.05 \\
.04\end{array}$ & $\begin{array}{l}.76 \\
.88 \\
.84 \\
.83\end{array}$ & $\begin{array}{l}.18 \\
.11 \\
.11 \\
.11\end{array}$ & $\begin{array}{l}.77 \\
.85 \\
.80\end{array}$ & $\begin{array}{l}.11 \\
.11 \\
.12\end{array}$ & $\begin{array}{l}.66 \\
.80\end{array}$ & $\begin{array}{l}.12 \\
.11\end{array}$ & .35 & .11 \\
\hline $\begin{array}{l}\text { Control } \\
\text { Group }\end{array}$ & $\begin{array}{r}9 \\
18 \\
27 \\
36 \\
45\end{array}$ & $\begin{array}{l}.95 \\
.72 \\
.76 \\
.76 \\
.77 \\
\end{array}$ & $\begin{array}{l}.02 \\
.05 \\
.06 \\
.05 \\
.06\end{array}$ & $\begin{array}{l}.33 \\
.55 \\
.67 \\
.62\end{array}$ & $\begin{array}{l}.09 \\
.09 \\
.04 \\
.04\end{array}$ & $\begin{array}{l}.35 \\
.39 \\
.37\end{array}$ & $\begin{array}{l}.08 \\
.06 \\
.08\end{array}$ & $\begin{array}{l}.13 \\
.30\end{array}$ & $\begin{array}{l}.05 \\
.08\end{array}$ & .07 & .03 \\
\hline
\end{tabular}


auditory signals into representations that permitted spatial inferences. Patterns were identified in imaginary two-dimensional space on the basis of auditory inputs that were unlikely to have been heard before. The subjects did not learn to attach a label to a set of dots and dashes in paired-associate fashion; rather they used rules to discover a spatial pattern. Instructions in the use of the patterns as a mnemonic enabled eight out of nine subjects to remember binary sequences far beyond the capacity of control subjects.

The finding that it was not significantly more difficult to identify rotated letters than upright letters does not weaken the case for imagery. It is true that Kolers and Perkins (1969) found that in visual perception of sequences of letters it is slightly more difficult to identify rotated letters than those in normal position. However, the low overall error rate in that study $(4 \%)$ as well as in the present one, makes it unrealistic to expect statistically significant differences related to orientation of letters. Unless special conditions are introduced to require internal rotation of images, differences in difficulty of simple identification are apt to be minimal (Cooper \& Shepard, 1973).

Three levels of information processing can be readily distinguished in the present experiments. At the sensory level, acoustic signals are discriminated as to membership in one of two classes. Second, at an abstract spatial level, the products of the sensory classification are accumulated in a two-dimensional array. Third, the verbal system provides a label for the pattern constructed in the spatial array.

The second level of processing raises interesting questions. Here the serial input is somehow stored so that a sequence can be treated as a chunk. It seems likely that parallel processing occurs at this stage, even though subjects may identify a letter on the basis of some distinctive feature that does not require apprehension of all nine positions in the array at the moment of discovery. In order to discover even a feature subject must hold several positions for simultaneous processing.

Simon (1969, Pp. $43 \mathrm{ff}$ ) has suggested that many presumed visual images are processed and stored serially as list structures. Thus, a three by three array could be stored in a computer program as three lists, each list containing three characters. While Simon's analysis may be reasonable for the matrices of decimal digits and letters that he investigated, the analogy with the computer suggests that at some microlevel sets of elements are processed in parallel. A nine-element set of binary elements is within the limit of a word (set of elements processed in parallel) for a computer and it is also within the immediate memory capacity of many humans.

Of interest in Experiment II is the concurrent storage by subjects of two different series of elements. A verbal storage held several letters, while during the same period a spatial storage accummulated binary signals until identification of a new letter. Previous studies of concurrent verbal activity have found concurrent storage of two independent strings of verbal symbols (Peterson, 1969). In the present experiment, experimental confirmation of the working of multiple storage systems in close cooperation is provided. Anderson and Bower (1973) have assumed several stores must work together in the perception of sentences. Experiment II supports the more general interpretation that multiple short-term storages function cooperatively as humans process information. The two storages in the present case are most appropriately considered as arising at different levels of information processing. The obvious fact that one storage retains information longer than the other does not warrant labeling one long and the other short. The difference in length of retention of information arises out of the needs of the task, i.e., subject must replace signals in the spatial array from one sequence of nine to the next, while the letters must be held until time of recall.

The technique seems promising for the objective study of subjective imagery. Numerous applications of the technique suggest themselves, for instance, the study of transformations of inputs into novel outputs by means of the spatial grammar. Hopefully the technique may add to the growing understanding of the working of cognitive structures in general.

\section{REFERENCES}

Anderson, J. R., \& Bower, G. H. Human associative memory. New York: Wiley, 1973.

Atwood, G. E. An experimental study of visual imagination and memory. Cognitive Psychology, 1971, 2, 290-299.

Bower, G. H. Mental imagery and associative learning. In $I$. W. Gregg (Ed.), Cognition in learning and memory. New York: Wiley, 1972.

Cooper, L. A., \& Shepard, R. N. Chronometric studies of the rotation of mental images. In W. G. Chase (Ed.), Visual information processing. New York: Academic Press, 1973.

Kolers, P. A., Perkins, D. N, Orientation of letters and errors in their recognition. Perception \& Psychophysics, 1969, 5, 265-269.

Paivio, A. Imagery and verbal processes. New York: Holt, Rinehart, \& Winston, 1971.

Peterson, L. R. Concurrent verbal activity. Psychological Review, $1969,76,376-386$.

Simon, H. A. The science of the artificial. Cambridge: MIT Press, 1969.

(Received for publication June 6, 1974, revision received August 5, 1974.) 